\title{
Obesity as a risk factor for the admission of COVID-19 patients into ICU: a systematic review and meta-analysis
}

\author{
C. Bunn, S. Al-Naimi, A. Rajic and F. Ibrahim \\ ${ }^{1}$ Life Sciences Directorate, University of Suffolk, Ipswich, Suffolk, UK
}

Coronavirus disease-2019 (COVID-19) is an infectious disease caused by the recently discovered Severe Acute Respiratory Syndrome Coronavirus 2. An increasing amount of evidence suggests that obese individuals are at a greater risk of severe respiratory disease, including the admission into intensive care unit (ICU) ${ }^{(1-2)}$. Various potential molecular mechanisms have been suggested to link obesity with severe outcomes of COVID-19 such as dysfunctional adipose tissue-derived stem cells, disturbed metabolic homeostasis and a dysregulated immune system ${ }^{(3)}$. The aim of this meta-analysis is to assess the magnitude of obesity as a risk factor for the admission of COVID-19 patients into ICU.

PubMed and Web of Science databases were chosen for the literature searches within this review. The following search strings were used: ("OBESITY") and ("COVID-19") and ("ICU"). Search terms and filters were decided upon using the PICO model to identify the patient or population (P), intervention/exposure (I), comparison (C) and outcome (O). P - Patients infected with COVID-19, I Individuals with obesity, $\mathrm{C}$ - Obese to Non-obese individuals and $\mathrm{O}$ - Admission into ICU. The reviewing process included all studies that reported the presence of obesity and the specific outcomes of COVID-19 patients (i.e. the use of IMV OR the admission into ICU). Studies with free full access that were published in the year 2020 were eligible for inclusion. Only retrospective cohort studies were included. The study was conducted following the recommendations of the PRISMA) statement. WHO categories of BMI were used for to separate obese and non-obese patients. A meta- analysis was conducted on 23 retrospective studies published in the year 2020. A total of 14,180 participants across 9 different countries were included. Comparisons were carried out on the ICU admission rate between obese and non-obese patients infected with COVID-19.

Obesity was found to significantly increase the risk of admission into ICU by $71 \%(\mathrm{OR}=1.71 \mathrm{CI} 1.54-1.89, \mathrm{P}=<0.00001)$ compared to non-obese patients infected with COVID-19. Indicating that a higher BMI is associated with increased COVID-19 disease severity and the need for intensive care in hospitals.

This study confirmed obesity as a significant risk factor for the admission of COVID-19 patients into ICU. Therefore, BMI should be considered as a predictive measure when assessing the risk of severe outcomes from COVID-19, and additional precautionary measures should be put into place upon admission of individuals with obesity into hospital with COVID-19. Moreover, such significant findings should encourage effective action from public health sectors and the government to raise awareness to the general population of the risks associated with obesity, and how these have been amplified by the COVID-19 pandemic, as well as uninvestigated existing and potential future infectious diseases.

\section{References}

1. Lighter, J., Phillips, M., Hochman, S., et al. (2020) Clinic Infect Dis, 71(15), 896-897.

2. Simonnet A, Chetboun M, Poissy J., et al. (2020) Obes (Silver Spring). 28(7), 1195-1199.

3. Ritter, A., Kreis, N., Louwen, F., et al. (2020) Int J Mol Sci, 21(16), 5793. 